\title{
Development of the Game Hangman in Assembly Programming Language
}

\author{
Stefan Tešanović and Predrag Mitrović
}

\begin{abstract}
This paper describes the realization of the Hangman game in Microsoft Visual Studio, using the assembler programming language, Kip Irvine's and MASM libraries. Knowledge of the work in the software tool is demonstrated, as well as advanced knowledge of assembler and work with library functions. The obtained game uses less CPU time than its realizations in other higher level programming languages.
\end{abstract}

Keywords - irvine32, masm, assembly, hangman, game

\section{INTRODUCTION}

$\mathrm{T}$ HIS paper provides an algorithm for the realization of popular computer game Hangman [1]. The basic idea of this work was the development of Hangman game using Microsoft Visual Studio 2015 programming environment and libraries used for easier implementation of the game in assembly programming language.

Assembler (assembly) language is a low-level programming language, and it is specific to a particular processor (microcontroller) architecture [2]. As the oldest programming language, and of all languages, it bears the closest resemblance to a native machine language. It provides a direct access to computer hardware, requiring you to understand much about your computer's architecture and operating system [3].

A large number of modern microcontrollers are based on a similar architecture as the microcontroller 8086 [3] and have similar abbreviations for the names of assembly instructions. So, the study of microcontroller 8086 is reckoned not only for pedagogical reasons but also for usability [4].

Through numerous examples of research being studied through lectures, students are led to a correct conclusion that when designing microcontrollers it is necessary to know the algorithm which the hardware will use and that it is necessary to know the hardware on which the software will be executed to write an optimal algorithm [2] - [8]. So, both parts of the design should be projected in parallel.

Today's software for digital signal processing is based on

Paper received May 29, 2018; revised November 25, 2018; accepted November 26, 2018. Date of publication December 25, 2018. The associate editor coordinating the review of this manuscript and approving it for publication was Prof. Nataša Nešković.

This paper is a revised and expanded version of the paper presented at the 25th Telecommunications Forum TELFOR 2017 [10].

Stefan Tešanović and Predrag Mitrović with School of Electrical Engineering, University of Belgrade, Bul. kralja Aleksandra 73, 11120 Belgrade, Serbia (e-mail: stefan@stefan.engineer, predrag.mitrovic@yahoo.com ). some high-level programming language, but optimization of code execution is achieved writing individual functions in assembly [9]. We can say that the assembly is used when we need fast and efficient processing of the signal.

The Irvine 32 library [3] makes programming in assembly easier and provides libraries for work with strings and support for MS-Windows features. Some of the main supporting features are graphical environment functions.

In this paper, we will describe designing the Hangman game using the $\mathrm{x} 86$ architecture with Irvine 32 libraries [3] in the programming environment Microsoft Visual Studio 2015.

The paper is organized as follows. In section II the $\mathrm{x} 86$ processor architecture is described in detail. Section III provides the game specification. In section IV are described functions and concepts used for developing the game. Concluding remarks are given in section $\mathrm{V}$.

\section{PROCESSOR X86 ARCHITECTURE}

Unlike programming in higher programming languages, where it is necessary to understand the endpoints of the procedural or object-oriented programming, for programming in assembler it is necessary to understand hardware.

Assembly language is not portable, because it is designed for a specific processor family. Thus, there are many different assembly languages widely used today, each based on a processor family [3].

Of all the hardware, the most important thing to us is the architecture of the central processor unit (CPU). In the CPU, where all calculations and logical operations take place, there are a limited number of storage locations named registers, a high-frequency clock, a control unit and an arithmetic logic unit [2] - [3].

In this paper, we have decided to use $\mathrm{x} 86$ processor for development of the project. In order to understand our realization of the game, it is necessary to get acquainted with the processor architecture, especially with registers and the way the strings are processed.

At our disposal, we have general-purpose registers. The general-purpose registers are primarily used for arithmetic and data movement. As shown in Fig. 1, the lower 16 bits of the EAX register can be referenced by the name AX. Portions of some registers can be addressed as 8-bit values. For example, the AX register has an 8-bit upper half named $\mathrm{AH}$ and an 8-bit lower half named AL [3].

Multiplication and division instructions automatically use EAX register. It is often called the extended accumulator register [2] - [3]. 


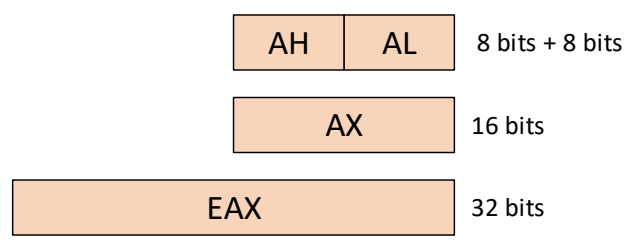

Fig. 1. General-purpose registers.

The same overlapping relationship that exists for the EAX registers also exists for EBX, ECX, and EDX registers.

High-speed memory transfer instructions use ESI and EDI registers. They are sometimes called the extended source index and extended destination index registers [2] $-[3]$.

These six registers represent the core of understanding how the instructions are executed in the assembler and the communication between the memory and the processor.

As we do the processing of strings in the project, it is necessary to emphasize that in the register ECX is the number of iterations of the corresponding instruction for working with strings.

\section{DESCRIPTION OF THE GAME REALIZATION}

The game is realized using the already known term which is to be guessed. For our purpose, we chose that the number of terms should be at least 10 . At the beginning of the game, the player tries to guess the term by writing letters from the standard input. If the entered letter does not exist in the specified term, then the part of Hangman is added. However, if the letter exists in the term which a player tries to guess, then the letter is written to the appropriate position in the word. If the letter has already been hit, the user is informed necessarily about it, and if the user by accident writes it, then it does not affect the game. Every time a new letter is entered from the standard input, it is necessary to print it on the screen. When a user guesses the current term, they are allowed to hit the new one. If the user misses the letters enough times, the entire Hangman is drawn out, and a player loses the game. The game can be interrupted by pressing the ESC button.

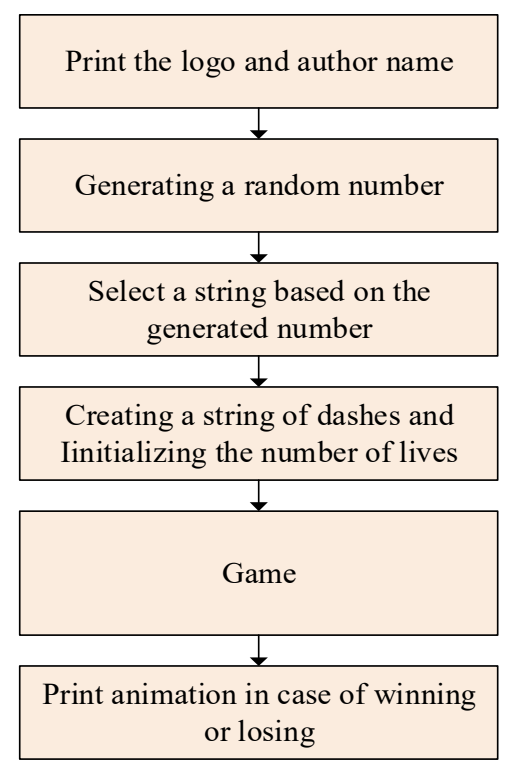

Fig. 2. A simplified program diagram.
The description of the project code and the principle of the game operation are described through the program flow diagram depicted in Fig. 2. In diagram from Fig. 2, each of the segments will be explained in separate subsections.

\section{A. Print the logo and name of the author}

At the very beginning of the program, we print the logo as a series of strings. A variable endl represents a string in which we typed two characters that represent the transition to a new line. In the variable messageSize, the length of this string is saved.

To print the string, we use the WriteConsole function [8]. It prints a series of characters on the screen starting from the current position of the cursor and moves the cursor to the position of the last printed character. In order for this function to be used before its use, it is necessary to take access to the standard input or output. With the GetStdHandle function [8], we take a standard input and output handle, which can then use some of the following functions: ReadFile, WriteFile, ReadConsoleInput, WriteConsole or GetConsoleScreenBufferInfo [8]. All mentioned functions can be found in Irvine libraries [3], [6] - [8].

\section{B. Generating a random number}

Generating a random number we have achieved the power of two Irvine functions [3], [6-8]. The Randomize function restarts the randomly generated number based on the current time. If we did not use this function, we would always have the same random number. The RandomRange function generates an unsigned pseudo-random 32-bit integer in the range 0 to $N-1$. In our case, it generates a number from 0 to 9 . The code that realizes this is shown in Fig. 3.

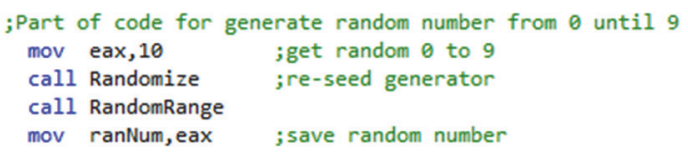

Fig. 3. Code generating a random number.

\section{Choosing the word which we guess}

Based on the randomly generated number, we need to select one of the ten words, which we have previously defined. Fig. 4 shows the declaration of the string of all the words that can be selected.

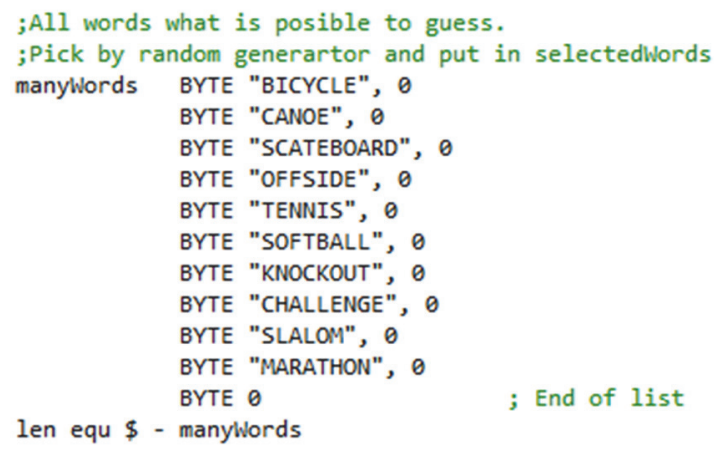

Fig. 4. Define words what to guess. 
the standard input is whether it is ESC. In case the ESC key is pressed, i.e., the entered character has a value of 27 , it is necessary to suspend the execution of the game. Otherwise, we continue to execute the program.

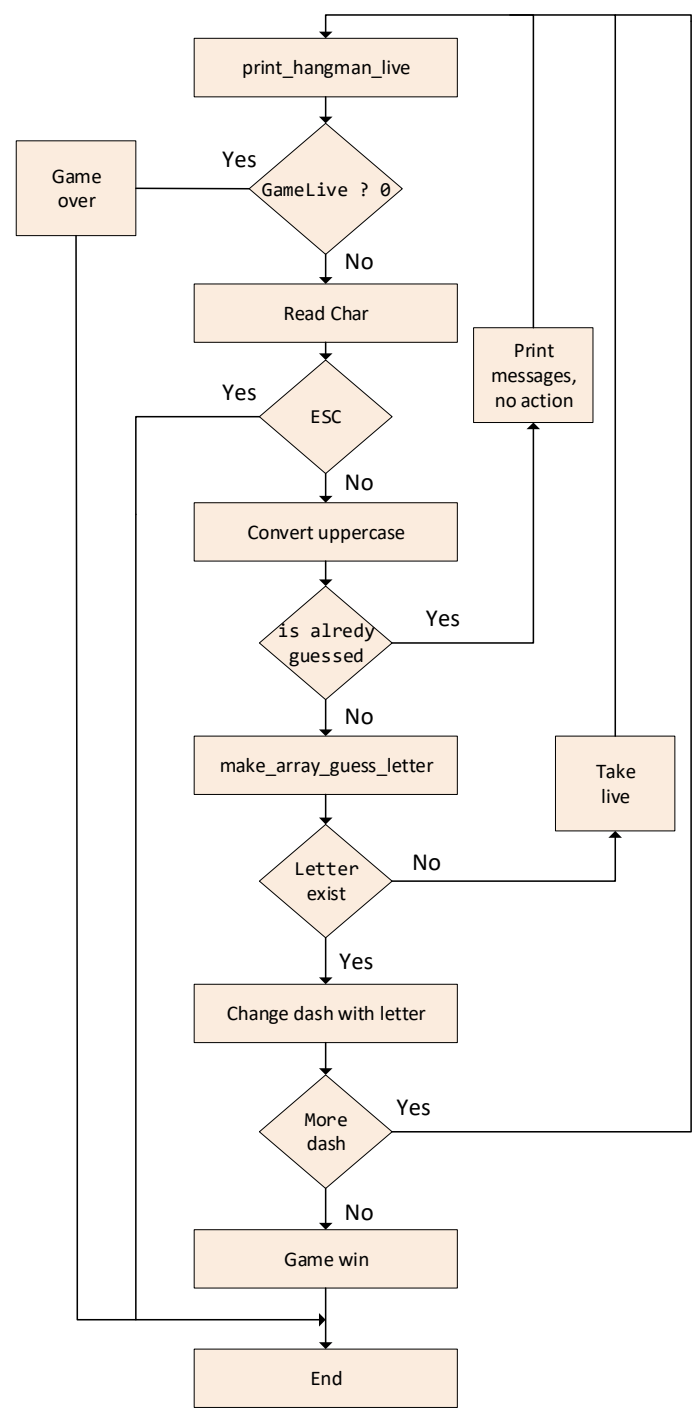

Fig. 9. Game flow diagram.

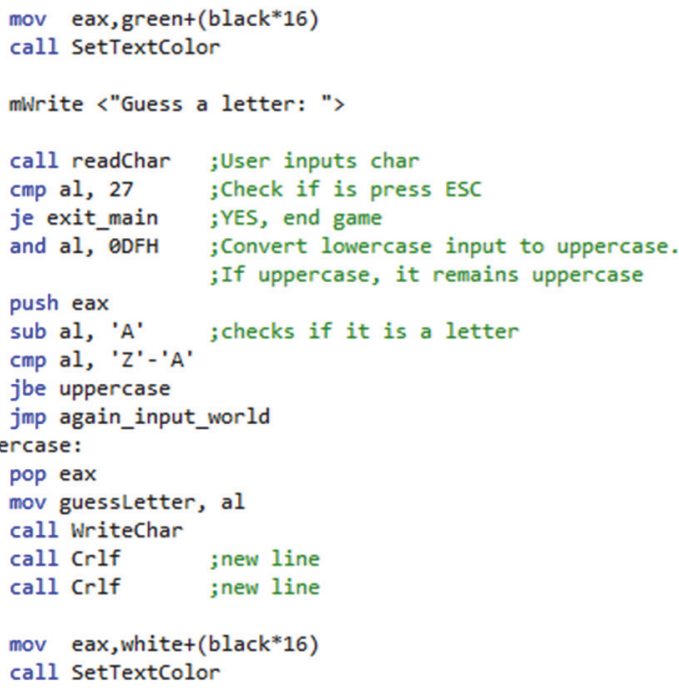

Fig. 10. Code for the letter loading realization.
Since the variables (terms) are consisting exclusively of capital letters, it is necessary to examine whether a large or a small letter is entered. If a small letter is entered, the program converts it to a capital letter. Also in this part of the program, a message is printed to enter the letter and the printout of the entered letter itself. The print is green in order to be more visible to the player.

Next, it is necessary to check whether the entered letter has already been hit or not. In the variable guessLetterArray, all letters entered from the standard input are stored. In case the letter has already been hit, it is necessary to jump loop_guess_letter_exists over the loop, print the corresponding message and return to the beginning to re-enter the letters. The code that prints the message to the standard output is shown in Fig. 11.

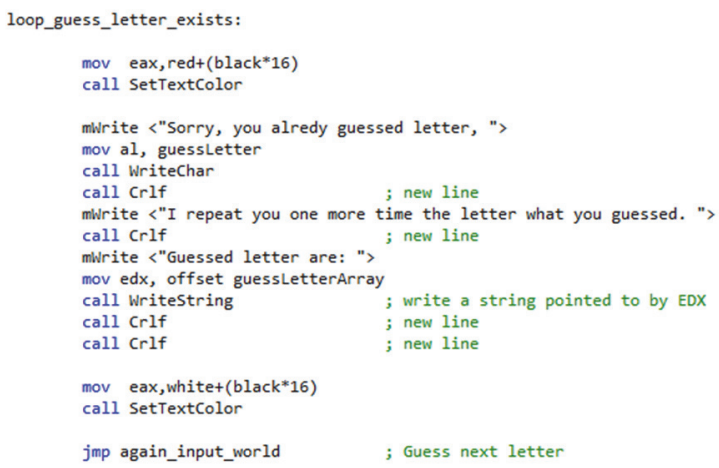

Fig. 11. Code for printing message that letter has already been hit.

In case the letter has not been hit yet, it is necessary to add it to the string guessLetterArray. This is accomplished by calling the make_array_guess_letter function. The function is shown in Fig. 12.

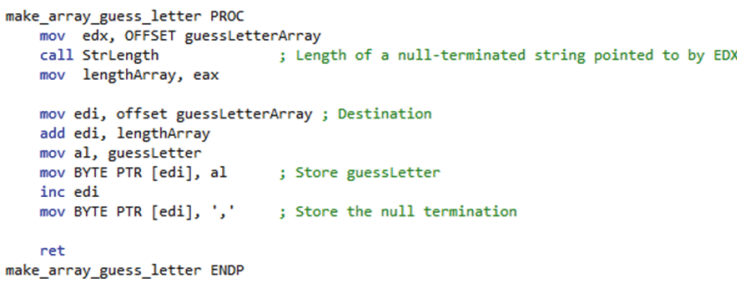

Fig. 12. Code to add letters entered into a string of entered characters.

It is also necessary to check if the entered letter is in the selected word, the selection of which is described in the chapter above. The search string is similar to a bit earlier and is shown in Fig. 13.

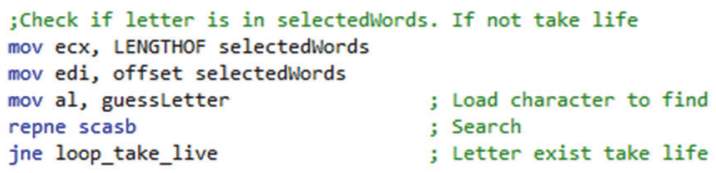

Fig. 13. Check whether the selected word contains the entered letter.

If the selected word does not contain the entered letter, we scroll loop_take_live over the loop, reducing the value of the variable statusGameLive to one, i.e., we take one life to the player and return to the beginning of that game. 
If the guess word contains an entered letter, then it is necessary to place the letter in the appropriate place in the guessWords strand, i.e., to replace the corresponding dash with letters in the same places as they are in selectedWords. The code that performs this realization is shown in Fig. 14.

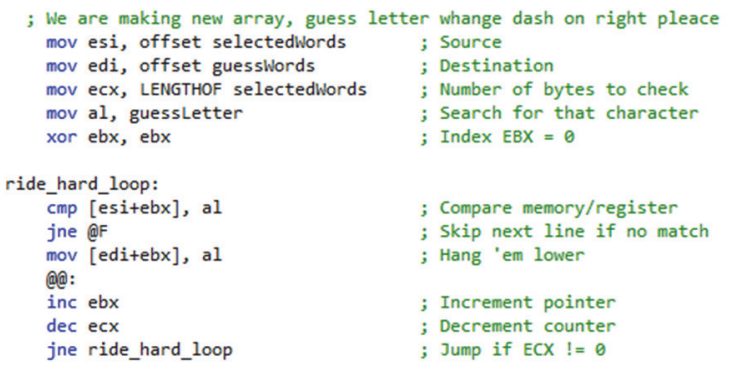

Fig. 14. Replace dashes with the letter inserted.

In the next step, we need to check if there are some other dashes in the guessWords strand or we hit all the letters. If guessWords contain dashboards, we return to the beginning of the game. Otherwise, we print the animation, and we end the game. The code for this realization is shown in Fig. 15.

\section{CONCLUSION}

In this paper, we describe a realization of the game Hangman. Although the game is well known and built numerous times, our realization gives an exciting approach to a programming assignment made entirely in assembly programming language. This realization is especially interesting for educational purposes because it provides an exciting way to introduce students to assembly programming and architecture of $\mathrm{x} 86$ processors.

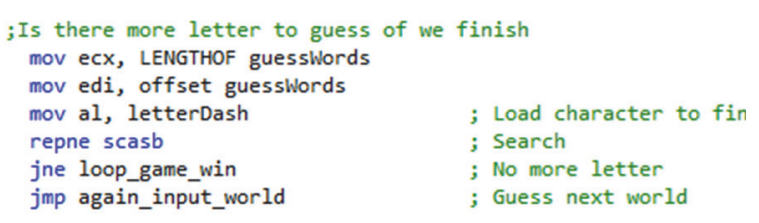

Fig. 15. Check if there are still unchecked letters.

\section{APPENDIX}

All codes of this realization can be downloaded from GitHub by following link https://goo.gl/cfaWH6.

\section{ACKNOWLEDGMENT}

We devote this work to professors, mentors $\mathrm{dr}$ Aleksandra Lekić and dr Milan Prokin, family and Giovanni who was always there for us with advice and support.

\section{REFERENCES}

[1] https://en.wikipedia.org/wiki/Hangman_(game)

[2] Carter, Paul A. PC Assembly Language. Lulu.com, 2007.

[3] Irvine, Kip R. Assembly language for Intel-based computers. Prentice Hall, 2003.

[4] Prokin, M. Računarska elektronika. Akademska misao, 2005

[5] Hyde, R. The art of assembly language. No Starch Press, 2010.

[6] http://kipirvine.com/asm/index6th.htm

[7] http://www.asmirvine.com/

[8] http://programming.msjc.edu/asm/help/index.html?page $=$ source $\% 2$ Fabout.htm

[9] Kuo, Sen M., Bob H. Lee, and Wenshun Tian. Real-time digital signal processing: fundamentals, implementations and applications. John Wiley \& Sons, 2013.

[10] S. Tešanović and P. Mitrović, Development of the Game Hangman in Assembly Programming Language, 2017 25th Telecommunications Forum (TELFOR), Belgrade, 2017, pp. 912915. 